\title{
BASED ON VISUAL BASIC DIFFERENTIAL WORKBENCH SYSTEM DESIGN AND IMPLEMENTATION OF MOTION
}

\author{
YanKai Liu ${ }^{1}$, MinLiang Zhang ${ }^{2}$ \\ ${ }^{1}$ Department of Mechanical Engineering, Shanghai University of Engineering Science, Shanghai 201600 China \\ ${ }^{2}$ Department of Mechanical Engineering, Shanghai University of Engineering Science, Shanghai 201600 China
}

\begin{abstract}
In this paper, we take the equipment precision differential bench which homemade by Shanghai University of Engineering and Technology for the study, and use the Visual Basic for precision motion system of differential table design and secondary development. Through experiments, we can get the change in position under different sports differential table, through the measurement of data analysis, we can get in the form of differential motion error table for further compensation error of the foundation.
\end{abstract}

Keywords: Visual Basic, Differential, The error analysis

\section{INTRODUCTION}

The increasing demand in the machining accuracy of precision mechanical products in the micro electronic technique, communication, spaceflight and biotechnology fields have stimulated the development of high-speed, high precision, complex, high-tech and environmental protection. The two-dimensional pecision workstations which are widely used in the mechanical design, manufacturing and processing, it will directly affect the development of precision machining technology. From a simple hardware to improve the accuracy of the work table is becoming increasingly difficult, on the one hand it will increase the cost of the device, on the other hand the equipment installation requirements are also increasing. From the above debate, we can control the device in terms of software, both at home and abroad, many scientists now study how to improve the accuracy and reduce the errors of table in software and hardware. The experimental subjects with precision / ultra-precision machining industry as the research background, the use of differential sampling theory of mechanical transmission and sampling error modeling errors combined ,analysis of the optimal speed range of servo motor .The quick differential of double benches can achieve the dynamic compensation of system error.

\section{RESEARCH STATUS AT HOME AND}

\section{ABROAD}

With the rapid development of modern computer technology, CNC machining technology and measurement technology, hardware and software technology has been rapid development. The combination between hardware and software have a more broad application prospects. Especially in China, with the rapid development of industry and the national economy, the quantity and quality of the machining center requirements are also increasing. So the study about the processing center software control technology will be more in-depth research, and the application will be more extensive.
The literature ${ }^{[1]}$ using two-frequency laser interferometer to measure dual screw error. According to certain time displacement of spacing, the computer measure the sampling error of the data processing and issue appropriate compensation signal. Through the appropriate error compensation interface is passed to the error of $\mathrm{CNC}$ machine tools $\mathrm{CNC}$ system consisting of $\mathrm{CNC}$ machine tools to complete the appropriate compensation. On any compensation point, in this way, the computer will be issued the compensation signal, which compensated by the CNC machine tools, until the end of travel. This method is similar to the real-time compensation, but this method is not suitable for high-speed movement. Therefore, we can design the software without compensation at high speed, then return to wait until the low speed compensation.

Samir Mekid worked at Compiegne Industrial University. $\mathrm{He}$ adopt the brushless DC motor to drive, hydrostatic bearing constitutes a precision linear slide. Also, he uses the method of internal model control to achieve a larger stroke nanopositioning. The precision stage of stroke reach $220 \mathrm{~mm}$, positioning accuracy can reach $16 \mathrm{~nm}$, the maximum speed of is $10 \mathrm{~mm} / \mathrm{s}$. The positioning of the table meet the requirements of the equipment. Seoul National University have developed a nano-micro-macro two positioning table. The macro-motion table uses a dynamic table sets ball screw nut mechanism and the micro-motion bench with PZT plus flexible hinge mechanism. The table adopts the method of double servo control, the position feedback of macro-motion table uses encoder and the position feedback of micro-motion table adopts a laser interferometer with a $10 \mathrm{~nm}$ accuracy, reaching to a $200 \mathrm{~mm}$ located route. The macro-motion can achieve great stroke and the high precision achieved by micro-motion. The positioning capabilities of the high precision drive mechanism positioning can make a compensation about the error which brought by macro-driven movement, reducing the system of macro-driven positioning accuracy requirements ${ }^{[2-4]}$. 
In China, universities and experts also conducted a precision bench-depth study, but the level of manufacturing machinery have a big gap between foreign and our country.

Tsinghua University is an important unit of domestic research precise positioning, they made a lot of achievements in research in areas such as precision table. Among them, the lithography Ultra Precision workstations studied by them, using flotation linear guides, linear motors and linear gratings consist a large stroke linear motion system and it can achieve more than $300 \mathrm{~mm}$ of travel, also you can make a micro-compensation about the precision of macro-table.A dual-positioning stage studied by Harbin Engineering University respectively using a linear motor and electric Primax microfilm shifters to achieve a large stroke and high precision positioning. The positioning system with a lower positioning accuracy can achieve a macro location and its micro-positioning by electrostriction microfilm displacement drive flexible workbench to achieve high positioning accuracy.It also can enable the system to get a higher positioning performance and brisk response speed and greatly reduced the structure of the system and so on ${ }^{[5-6]}$.

In this study,we can combine current research at home and abroad,starting from a realistic basic,constructed a sophisticated differential table.In the experiment,we take a measurement to the motion of the differenal table with the precision grating.By collecting motion data,we can get the differential motion table data and then make an analysis and comparison to the data.Finally,we lay the fundation for the analysis and compensation of the error.

\section{THE DESIGN OF DIFFERENTIAL MOVEMENT SYSTEM}

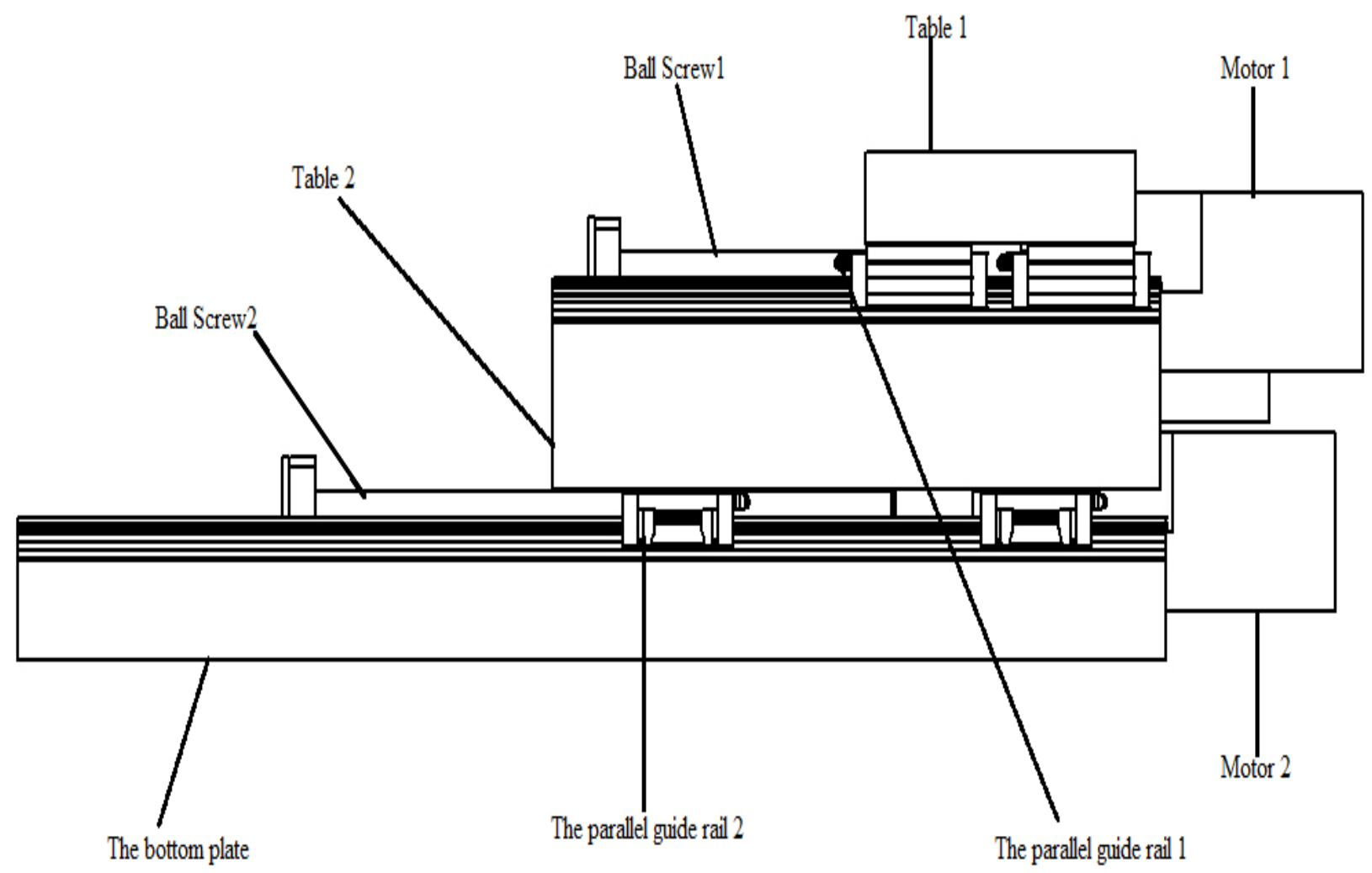

Fig-1: Chart table of the precision differential table

In the above-described mechanism, we take a parallel superposition with the two tables. The two motors respectively control the two platforms. The ball screw as the bridge of the center, turn the rotary motion of the motor into translational stage. During the motion, the motion control card can play a role in the differential movement of the two tables.

Traditional micro-feed systems usually meet single technical indicator, but it cannot be widely used in practical engineering. We have finished many works in this research.
We have developed a set of differential micro-feeding experiments bench system. In the process of system error compensation study, we fully analyze the micro-feeding bench errors. It can provide theoretical guidance for China's traditional manufacturing industries.

Micro-feed system is a very complex system which includes the development of workbench, error acquisition, analysis, and error compensation research methods of the positioning system. In this paper, we take an analysis and comparison about the current large stroke ultra-precision positioning 
systems. Taking into account the actual processing required, we determine the motion control card, AC servo motor, linear encoders and differential test bench design. The motion control card is seen as the control system core; The AC servo motor is seen as the driver; The linear encoders are used for data monitoring, collection and feedback. The differentional mechanism experiment is seen as the end table. Among them, the differential micro-feeding system which is similiar to an double-drive means can achieve a high positioning precision. It uses the two-stage coarse positioning accuracy but it can achieve higher positioning accuracy through a two-stage differential control. In this paper, we take the micro-precision positioning. feed system as the study. Also we take the loe-cost,large stroke, highspeed and high-precision as the main indicators. Finally, we improve the positioning accuracy of the system for the primary purpose.

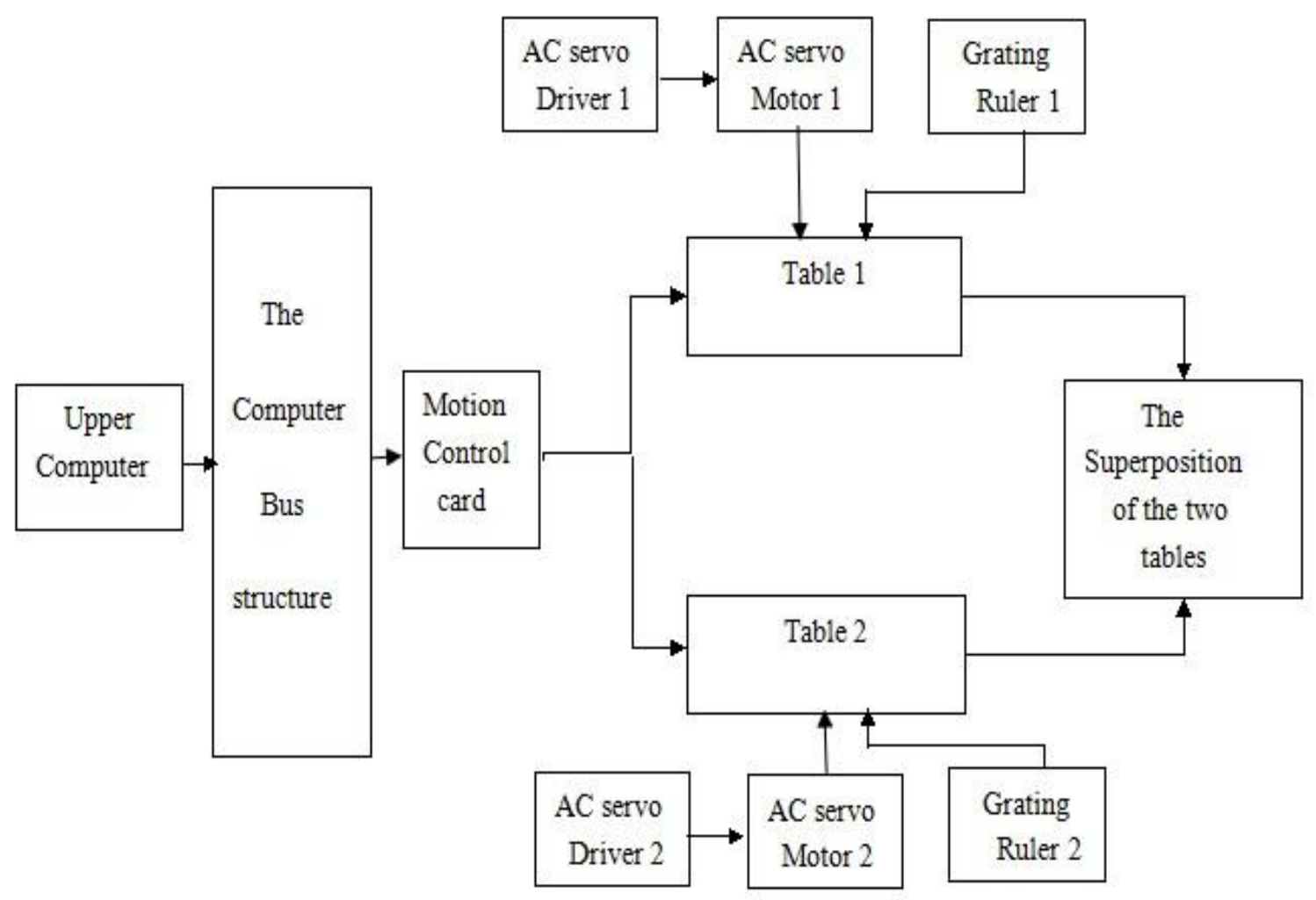

Fig-2: The system control chart of precision differential table.

The precision differential table is made up of two tables which were superimposed up and down.It resembles coarse and fine machining table.The system uses a precision grating as a precision differential table data acquisition unit, which monitors the location of the table in real time and timely feedback to the computer system. The AC servo motor which we used is Yaskawa SGMJV rotary motor.As a key part of the mechanical structure and motion control card, it carries the function following the instructions of the motor rotation motion into linear motion table institutions and feedback pulse data. PC and motion control card is the "Brain"of motion control system. On one hand, it monitors and sends instructions in real-time. On the other hand, it is responsible for receiving the feedback signal which produced by AC servo motor end pulse encoder end and mechanical transmission Linear and makes a comparative analysis.

\section{THE DESIGN OF SOFTWARE ABOUT THE DIFFERENTIAL MOTION MICRO-FEEDING SYSTEM}

In order to obtain the data of the accurate differential motion table,we have made secondary development about Galil Tools-Lite software which we buyed.Based on Visual Basic programming language to develop micro differential feed system design man-machine interface.According to the actual demand, we designed the communication connection, file editing, program editor, assistant editor, parameter setting, data feedback, data sampling and image display eight modules.In this paper, the development of the differential decline feed system man-machine interface has three main functions, namely the basic functions, editing and display functions.By the above design and development, we can easily obtain the data about the precision of the differential motion table. 


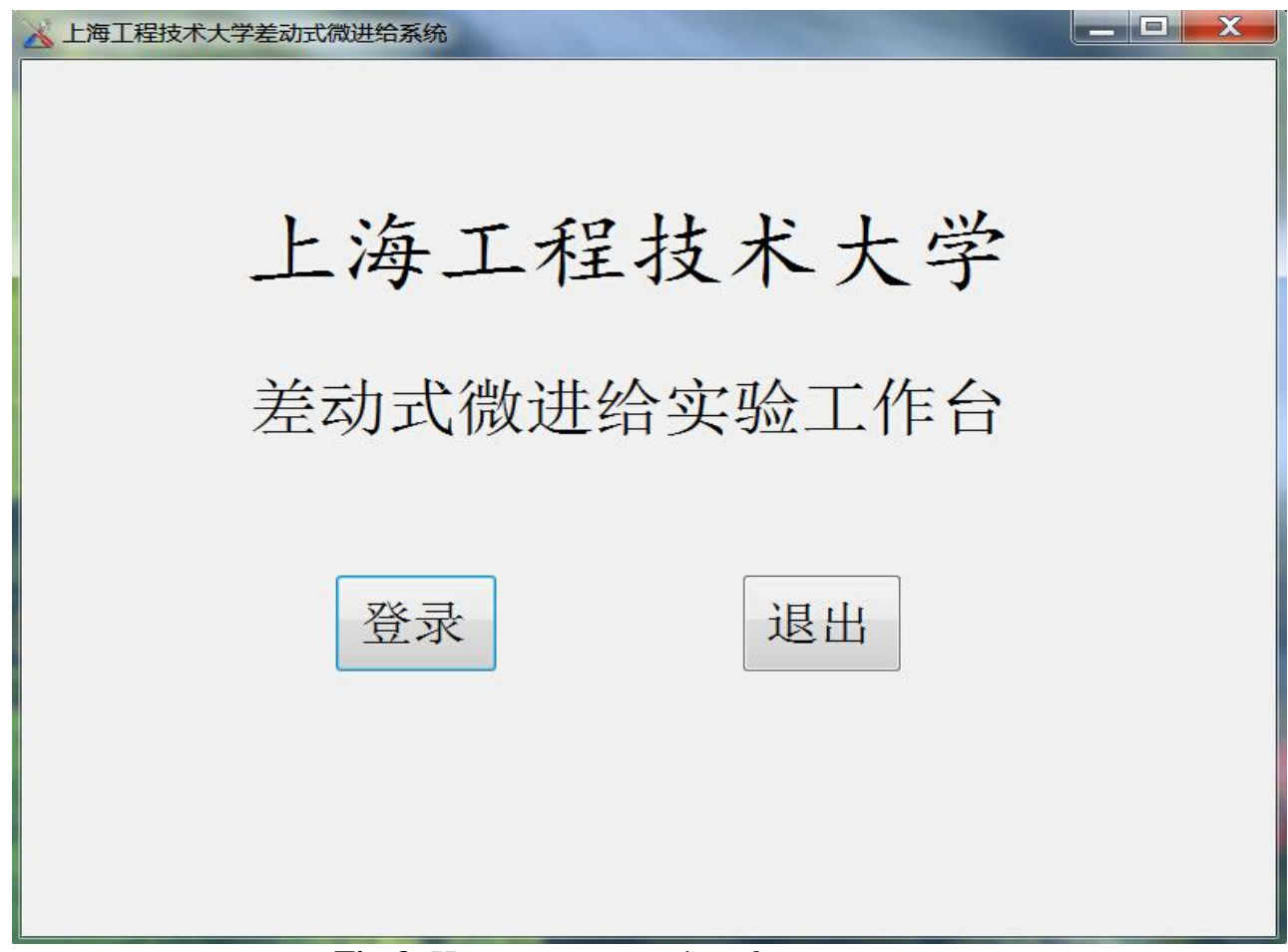

Fig-3: Human-computer interface entry page

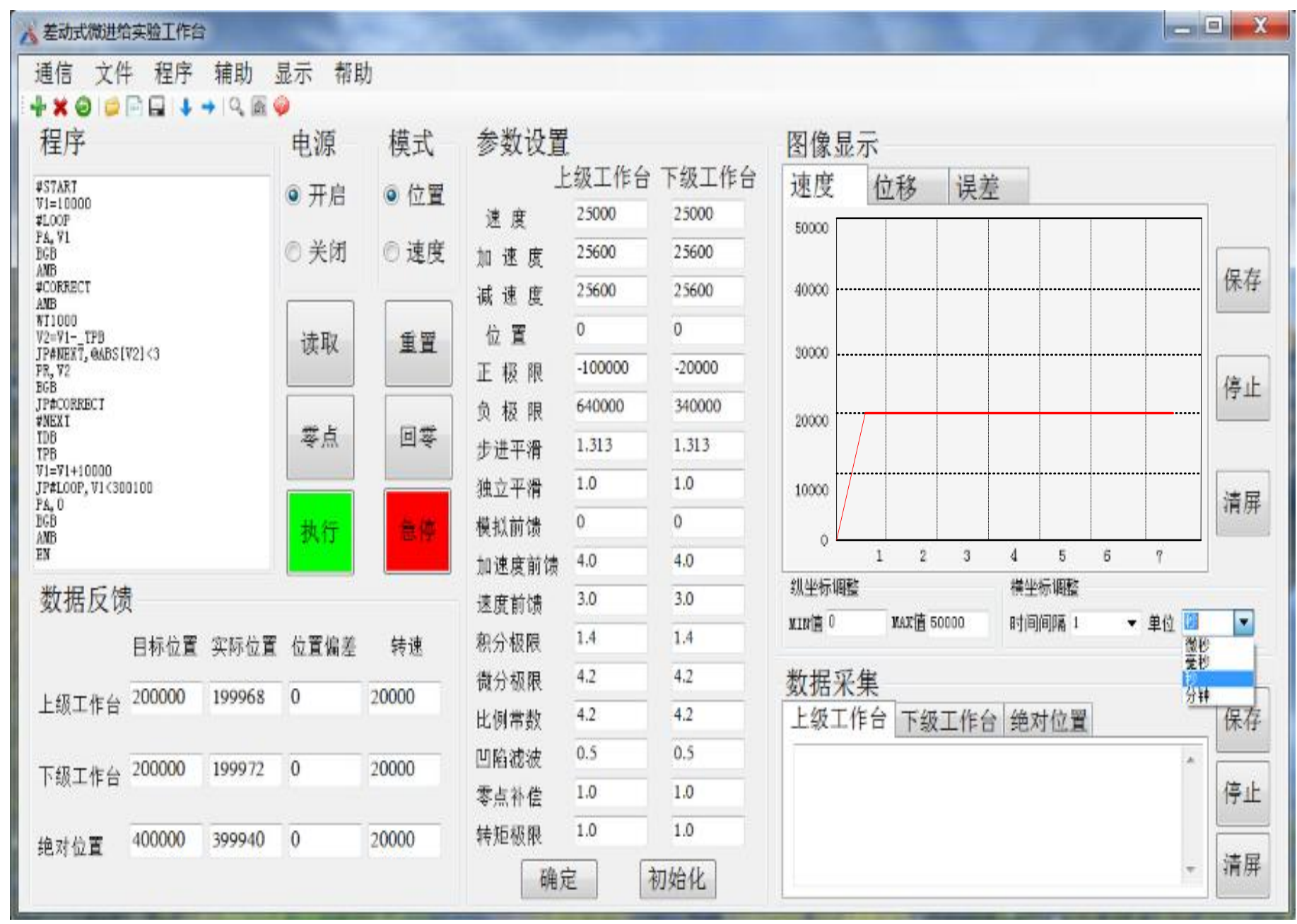

Fig-4: Human-computer main interface 
The interface with a relatively simple screen can feedback important parameters of each axis required by the user.It can dynamically collect feedback data, and shows the state of the image which owned the feeding system of the tables.It also can enables the communication system, program editing, image display, feedback data acquisition functions

\section{CONCLUSION}

Based on Mechanical Engineering, Shanghai University of Engineering Science's own test equipment precision differential bench for the study,we make an analyse about the differential form for precision bench.We design and analyze the motion of the two-dimensional table movement under the differential form with the help of Visual Basic.We measure and read the precision stage movement velocity, displacement, and an error,but the errors will inevitably be occured in the experiment.We also make an analysis and compensation about the error within engineering permissible error range.We further analysis and make an compensation about the error, which will has great theoretical analysis and practical effect for the China's manufacturing industry.Based on the platform of research and discovery, we can carry out further research on this error and structural precision differential table, such as the establishment of a database error, design three-dimensional sculpture and so on.

\section{ACKNOWLEDGEMENTS}

The authors would like to thank the financial support from Scientific Research Innovation Fund of Shanghai University of Engineering Science and the school's lab conditions to this research.

\section{REFERENCES}

[1] WeiHong Zhong,Baoguo Guan.The positioning error measurement and composition of CNC machine tool by laser interference.Modular Machine Tool \& Automatic Manufacturing Technique.2000.9

[2] Quan Li X-Y table friction modeling and simulation research[M].ShanDong University.2005.04

[3] DeJiang Zhang Intelligent control technology present situation and prospect[J].Journal of changchun university of technology.2002.08 58-61

[4] ChunYang Chang The review of intelligent control $[\mathrm{J}]$.Science and technology innovation herald.2012.12

[5] PuYang Jiang Based on expert system and intelligent control of circulating fluidized bed boiler[J].Industrial Technology 2011

[6] ChangZheng Huang,Yu Zhu. The review of intelligent control system [J]ShaoGuan University Journal 2001.03

\section{BIOGRAPHIES}

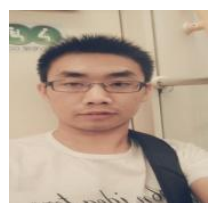

YanKai Liu is currently a master in the Mechnical design manfacturing and automation at Shanghai University of Engineering Science. His main subjects include product design, electronical and manufacturing.

\section{E-mail:15021998387@163.com}

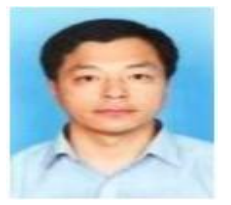

MinLiang Zhang is currently a professor ,vice President of the institute of mechanical engineering at Shanghai University of Engineering Science.

E-mail:Jx9988@163.com 